\title{
Survival and clinical outcomes of diabetic peripheral artery disease patients following a pain-free home- based walking program
}

\author{
Nicola Lamberti' ${ }^{1}$, Elpiniki Tsolaki ${ }^{2}$, Franco Guerzoni ${ }^{3}$, Nicola Napoli ${ }^{3}$, Luca Traina ${ }^{2}$, Giovanni Piva ${ }^{4}$, \\ Vincenzo Gasbarro ${ }^{2}$, Paolo Zamboni ${ }^{5}$, Sofia Straudi ${ }^{1,6}$, Roberto Manfredini ${ }^{7}$, Fabio Manfredini ${ }^{1,6}$ \\ 'Department of Neuroscience and Rehabilitation, University of Ferrara, Ferrara 44121, Italy. \\ ${ }^{2}$ Unit of Vascular and Endovascular Surgery, University Hospital of Ferrara, Ferrara 44124, Italy. \\ ${ }^{3}$ Department of Medical Statistics, University Hospital of Ferrara, Ferrara 44124, Italy. \\ ${ }^{4} \mathrm{PhD}$ program in Sustainability Environmental and Wellbeing, University of Ferrara, Ferrara 44121, Italy. \\ ${ }^{5}$ Vascular Diseases Center, University of Ferrara, Ferrara 44124, Italy. \\ ${ }^{6}$ Unit of Physical and Rehabilitation Medicine, University Hospital of Ferrara, Ferrara 44124, Italy. \\ ${ }^{7}$ Department of Medical Sciences, University of Ferrara, Ferrara 44121, Italy.
}

Correspondence to: Dr. Nicola Lamberti, Department of Neuroscience and Rehabilitation, University of Ferrara, Via Luigi Borsari 46, Ferrara 44121, Italy. E-mail:Imbncl@unife.it

How to cite this article: Lamberti N, Tsolaki E, Guerzoni F, Napoli N, Traina L, Piva G, Gasbarro V, Zamboni P, Straudi S, Manfredini R, Manfredini F. Survival and clinical outcomes of diabetic peripheral artery disease patients following a pain-free home-based walking program. Vessel Plus 2022;6:9. https://dx.doi.org/10.20517/2574-1209.2021.79

Received: 28 May 2021 First Decision: 18 Jun 2021 Revised: 2 Jul 2021 Accepted: 9 Aug 2021 First online: 17 Feb 2022

Academic Editors: Giuseppe Biondi-Zoccai, Elena Cavarretta, Alexander D. Verin Copy Editor: Yue-Yue Zhang Production Editor: Yue-Yue Zhang

\section{Abstract}

Aim: We retrospectively examined the impact on the rate of survival of pain-free home-based exercise in diabetic peripheral artery disease patients compared to patients receiving usual care.

Methods: In total, 202 patients at Fontaine's Stage II with diabetes were studied. Half were enrolled in a structured home-based exercise program (E), whereas the other half received walking advice as the active control group (C). Long-term clinical outcomes at five years were gathered from the Emilia-Romagna Health Service Registry, with survival probability selected as the primary outcome.

Results: At baseline, the two groups did not differ for any demographic or clinical characteristics. High adherence to the program was recorded in Group E ( $88 \%$ of home-walking sessions executed, with an average distance walked during the program of $174 \mathrm{~km}$ ). After five years, a survival rate of $90 \%$ for Group $\mathrm{E}$ and $60 \%$ for Group C was observed, with a significantly $(P<0.001)$ higher mortality risk for Group C [Hazard ratio $(H R)=3.92]$. 
Additionally, among secondary outcomes, Group E showed a significantly $(P=0.048)$ lower rate of peripheral revascularizations than Group C (15\% vs. 24\%, respectively; $H R=1.91)$, all-cause hospitalizations $(P=0.007 ; 61 \%$ vs. $80 \%, H R=1.58$ ), and amputations ( $P=0.049 ; 6 \%$ vs. $13 \%, H R=2.47$ ). In a Cox multivariate-proportional regression model of the entire population, the predictors of survival probability were age $(H R=1.05), C$ harlson index $(H R=1.24)$, lower ankle-brachial index $(H R=6.66)$, and control group $(H R=4.99)$.

Conclusion: A simple sustainable program aimed at improving mobility of diabetic patients with claudication at high cardiovascular risk was associated with better survival and long-term clinical outcomes.

Keywords: Exercise therapy, peripheral arterial disease, diabetes, rehabilitation, mortality

\section{INTRODUCTION}

Atherosclerotic peripheral artery disease (PAD) is a highly prevalent and undertreated global disease, with a negative association with premature cardiovascular events and death ${ }^{[1]}$. Atherosclerotic lesions in the arteries of the lower limbs result in impaired blood flow to the legs of patients who experience cramping pain during walking and a limitation of their daily activities ${ }^{[2-3]}$.

In patients with diabetes, PAD is highly prevalent ${ }^{[4]}$, and it represents the most common manifestation of cardiovascular diseases. Indeed, PAD in diabetic subjects reflects a systemic atherosclerotic disease with peculiar characteristics, such as a high incidence of vascular calcifications ${ }^{[5]}$, rapid progression toward more severe PAD stages, and prevalent involvement of distal and bilateral arteries ${ }^{[5]}$. All these factors are ultimately associated with worst long-term clinical outcomes ${ }^{[4,6-7]}$ including a high risk of amputations ${ }^{[8]}$, unfavorable cardiovascular outcomes, and higher mortality in diabetic PAD patients as compared to those in non-diabetic PAD patients ${ }^{[9-10]}$.

The first line of treatment in these individuals is lifestyle modification and exercise therapy ${ }^{[1]}$, with supervised treadmill exercise performed at an intensity such as to evoke moderate to severe pain as a firstline recommendation for patients with PAD and intermittent claudication ${ }^{[12]}$.

However, the presence of pain during walking might reduce adherence to programs ${ }^{[12]}$, as do other barriers such as costs, travel, and access. The presence of diabetes may also attenuate improvements in walking performance in patients with PAD following exercise ${ }^{[11]}$. Moreover, repeated cycles of exercise-induced ischemia followed by reperfusion may be associated with signs of muscular damage, an increase in inflammatory markers and reactive oxygen species production, and structural and metabolic changes in the muscle with reduced strength and function ${ }^{[13-16]}$.

In addition, patients with type 2 diabetes need to be carefully checked and monitored before engaging in an exercise program ${ }^{[7]}$. In particular, patients severely detrained should start exercise at low intensity with close supervision by healthcare professional, aiming at integration of regular physical activity into daily routine $\mathrm{e}^{[7]}$. However, regardless of exercise length and modality, regular intensive walking exercise in more effective than usual care ${ }^{[17]}$.

More than a decade ago, a structured low-intensity, pain-free program prescribed at hospital and executed at home was developed and introduced into clinical practice for patients with $\mathrm{PAD}^{[18-20]}$. The Test in-Train out program (TiTo) proved itself to be effective on functional capacity and quality of life in patients with claudication, even in the presence of comorbidities ${ }^{[2,22]}$, when compared to surgical revascularization ${ }^{[23]}$, 
with favorable long-term clinical outcomes ${ }^{[24-25]}$. The program was also successfully translated to stroke survivors, patients with end-stage kidney disease, and people with multiple sclerosis ${ }^{[26-30]}$.

Therefore, given the high risk of mortality reported in diabetic PAD patients and the protective effect of exercise programs, we hypothesize that a different risk of mortality rates at five years would be observable in diabetic PAD patients enrolled or not into an exercise program.

The aim of this retrospective study was to examine the rate of survival, hospitalizations, and lower extremity amputations in a cohort of diabetic PAD patients enrolled in the TiTo home-based exercise program compared to another matched cohort of diabetic PAD patients acting as a control group.

\section{METHODS}

\section{Study design and setting}

This single-center cohort study was conducted at University Hospital of Ferrara, Italy, between 2013 and 2014. The study was approved by The Local Ethics Committee (CE-AVEC) (approval number 277/2019), but written informed consent was not attainable from all participants.

This study retrospectively analyzed a prospectively collected database of patients with PAD who were recruited at Unit of Vascular Surgery and referred for the training program at the Unit of Rehabilitation Medicine. A follow-up period of five years was considered. Long-term outcomes were gathered from the Emilia-Romagna Regional Health Service Registry.

\section{Subjects}

A cohort of diabetic PAD patients were enrolled in the rehabilitation program. The included patients presented with PAD at Leriche-Fontaine Stage II; diagnosis of type 2 diabetes with habitual use of oral hypoglycemic agents, insulin, or both; and no contraindications to low-intensity exercise training (heart failure, amputations, severe cognitive impairments, etc.).

During the same temporal frame, a parallel control group of patients with PAD with the same inclusion criteria were also studied from those screened at the outpatients' clinics of the Department of Vascular Surgery.

Patients were then classified into two groups according to the study objectives: exercise (E) and control (C).

Data regarding clinical status (including medication, risk factors, and comorbidities), PAD severity (including type of lesions), and ankle-brachial index were collected from the medical records of each patient. An independent researcher blinded to the group allocations of the patients created the dataset with the clinical information.

\section{Exercise group: training program}

All patients received the "Test in-Train out" home-based pain-free exercise program ${ }^{[19,20]}$.

This low-intensity structured exercise program was prescribed during approximately monthly visits at the hospital, and it was executed at home. The program is scheduled with two daily 10 min sessions of intermittent walking (with a 1:1 walk: rest ratio) at a prescribed speed. This training speed, which is converted into a walking cadence (steps/min) and maintained at home by the use of a metronome, was slower than the individual's walking speed at the beginning and progressively increased weekly. A daily log 
to monitor the program execution was provided to the patients, and feedback was requested at each hospital visit. The program lasted 6-12 months. Patients were discharged when they perceived a satisfactory upgrade of mobility, and a stable improvement in the pain-free walking distance was attained (e.g., when they reached a measured symptom-free walking speed of normal for sex and age, which was confirmed in two subsequent tests). Discharge was also possible when no changes in walking ability were observed after repeated measurements (e.g., for intercurrent osteoarticular pathologies) or for the impossibility or willingness to exercise or to complete the program. More details on the exercise program are reported elsewhere $^{[19,20]}$.

\section{Control group}

Patients in the control group who underwent duplex examination without functional assessment were instructed to maintain a healthy and active lifestyle, according to the TASC II guidelines ${ }^{[31]}$. In detail, patients were advised to perform track walking of sufficient intensity to bring on claudication, followed by rest, over the course of a 30-60 min session, three times per week ${ }^{[31]}$. A 12-month follow up visit was also scheduled with instrumental and clinical examinations, without intermediate visits.

After discharge, patients of both groups received recommendations to perform physical activity and to maintain an active and healthy lifestyle, according to the guidelines ${ }^{[31]}$.

\section{Study outcomes}

Five-year survival probability was the primary outcome. Secondary outcomes included PAD-related lower limb revascularization (including both endovascular and surgical procedures), all-cause hospitalizations, and lower limb amputations (minor or major). Outcomes were considered after the date of the entrance of participants into the program for Group E and after the vascular surgeon's visit for Group C. A five-year follow up period was obtained for all participants; in the case of death in absence of a precise outcome (e.g., amputation), data were censored at the date of death.

\section{Statistical analysis}

Data distribution was verified with a Kolmogorov-Smirnov test. Differences in baseline characteristics for the two groups were evaluated with Chi-squared tests, Student's $t$-tests, or Mann-Whitney tests, as appropriate.

Kaplan-Meier estimates of the distribution of time from enrollment to date of death and a log-rank test for trend were used to compare the curves of the patient group.

Multivariate Cox proportional hazards regression analyses were employed to analyze the effect of several predictor variables on the primary outcome for each group. Because of the limited number of events, multivariate HRs were calculated using a forward approach, with an entry limit of $P<0.05$. A $P$ value $<0.05$ was considered statistically significant.

All statistical analyses were performed using MedCalc Statistical Software version 19.8 (MedCalc Software bvba, Ostend, Belgium).

\section{RESULTS}

The clinical records of 416 claudication patients who visited the Department of Vascular Surgery between 2013 and 2014 were analyzed. In total, 196 patients underwent the rehabilitation program, and the remaining patients underwent vascular screening. In total, 214 patients were excluded because they were 
not diagnosed with diabetes $(n=187)$ or did not comply with the other inclusion criteria $(n=27)$. The final sample included 202 diabetic patients with claudication, 101 belonging to Group E and 101 to Group C [Figure 1].

At baseline, the two groups did not differ for any demographic or clinical characteristics or in PAD severity [Table 1].

In addition, the two groups were also balanced for duration of diabetes (E, $5 \pm 4$ years $v s$. $C, 5 \pm 5$ years; $P=$ 0.79 ) and for the proportion of patients with insulin-dependent diabetes (E, 39\% vs. C, $41 \% ; P=0.58)$.

\section{Exercise group}

In total, 98 patients in Group E safely completed the training program without any adverse events related to the training; the remaining three patients dropped out for health reasons. The program lasted for a mean of $9 \pm 1$ months, with a high adherence (mean value of $88 \%$ of home-walking sessions executed with respect to the prescribed sessions) and a mean distance walked at home of $174 \pm 29 \mathrm{~km}$. Seventy-five patients completed $100 \%$ of the training sessions prescribed, while the remaining twenty-three patients performed at least $60 \%$ of the program.

\section{Primary outcome: survival probability}

In the following five years after the baseline data collection, 49 (24\%) deaths occurred, 9 in Group E and 40 in Group C, corresponding to a survival rate of $90 \%$ for Group E and 60\% for Group C.

Kaplan-Meier analysis [Figure 2] confirmed a significantly $(P<0.001)$ higher mortality risk for Group C as compared to that for Group E, with an HR of 3.92 [95\% confidence interval (CI) 2.24-6.87].

\section{Secondary outcomes}

A between-group significant difference (log-rank $P=0.048$ ) was also observed for PAD-related revascularizations, with 15 positive cases in Group E and 24 positive cases in Group C, with a HR of 1.91 (95\%CI: 1.01-3.62) [Figure 3A].

Additionally, for all-cause hospitalizations, a significant difference was found (log-rank $P=0.007)$, with 61 hospitalizations in Group E vs. 79 in Group C and an HR of 1.58 (95\%CI: 1.13-2.21) [Figure 3B].

Finally, amputations showed a significant difference (log-rank $P=0.049$ ), despite the few positive cases ( 6 in Group E and 13 in Group C), with a corresponding HR of 2.47 (95\%CI: 1.00-6.10) [Figure 3C].

A sub-analysis focusing on only hospitalizations for cardiovascular diseases confirmed the previously observed difference for all-cause hospitalizations; in particular, 43 events were found in Group E compared to 64 events in Group C [log-rank $P=0.031 ; \mathrm{HR}=1.47$ (95\%CI: 1.07-2.12)].

\section{Predictors of survival probability}

Multivariate Cox proportional hazard regression models highlighted that age [HR $=1.05$ (95\%CI: 1.01 1.09)], Charlson index [HR $=1.24$ (95\%CI: 1.02-1.50)] indicating the presence of comorbidities, $\mathrm{ABI}$ of the more impaired limb representing PAD severity $[\mathrm{HR}=6.66(95 \% \mathrm{CI}: 1.37-32.05)]$, and control group $[\mathrm{HR}=$ 4.99 (95\%CI: $2.40-10.40)]$ were the only predictors of mortality in the entire population. 
Table 1. Baseline comparison of the two study groups

\begin{tabular}{|c|c|c|c|}
\hline & Exercise $(n=101)$ & Control $(n=101)$ & $\mathbf{P}$ \\
\hline Age, years & $69 \pm 10$ & $69 \pm 11$ & n.s. \\
\hline Males, $\mathrm{n}(\%)$ & $65(66)$ & $70(71)$ & n.s. \\
\hline \multicolumn{4}{|l|}{ Risk factors; $n(\%)$} \\
\hline Smoking & $86(85)$ & $86(85)$ & n.s. \\
\hline Hypertension & $80(79)$ & $78(77)$ & n.s. \\
\hline Hyperlipidemia & $71(70)$ & $63(62)$ & n.s. \\
\hline Diabetes mellitus & $101(100)$ & $101(100)$ & n.s. \\
\hline Chronic kidney disease & $34(34)$ & $33(33)$ & n.s. \\
\hline \multicolumn{4}{|l|}{ Comorbidities; n (\%) } \\
\hline Ischemic heart disease & $30(30)$ & $26(26)$ & n.s. \\
\hline Stroke & $10(10)$ & $15(15)$ & n.s. \\
\hline Pulmonary disease & $11(11)$ & $18(18)$ & n.s. \\
\hline Neoplastic disease & $10(10)$ & $18(18)$ & n.s. \\
\hline Age-adjusted charlson index & $7 \pm 2$ & $8 \pm 2$ & n.s. \\
\hline \multicolumn{4}{|l|}{ Laboratory values } \\
\hline Hemoglobin, g/dL & $13.5 \pm 1.4$ & $12.6 \pm 1.8$ & n.s. \\
\hline Total cholesterol, mg/dL & $202 \pm 69$ & $174 \pm 50$ & n.s. \\
\hline Triglycerides, mg/dL & $178 \pm 98$ & $152 \pm 81$ & n.s. \\
\hline Serum creatinine, $\mathrm{mg} / \mathrm{dL}$ & $1.45 \pm 0.89$ & $1.54 \pm 1.07$ & n.s. \\
\hline \multicolumn{4}{|l|}{ Peripheral artery disease } \\
\hline Lower limbs revascularization & $15(10)$ & 19 (19) & n.s. \\
\hline Bilateral disease & $82(81)$ & $79(78)$ & n.s. \\
\hline$A B I$ more impaired limb & $0.63 \pm 0.16$ & $0.61 \pm 0.19$ & n.s. \\
\hline ABI less impaired limb & $0.85 \pm 0.20$ & $0.78 \pm 0.19$ & n.s. \\
\hline
\end{tabular}

ABI: Ankle-brachial index; n.s.: not significant.

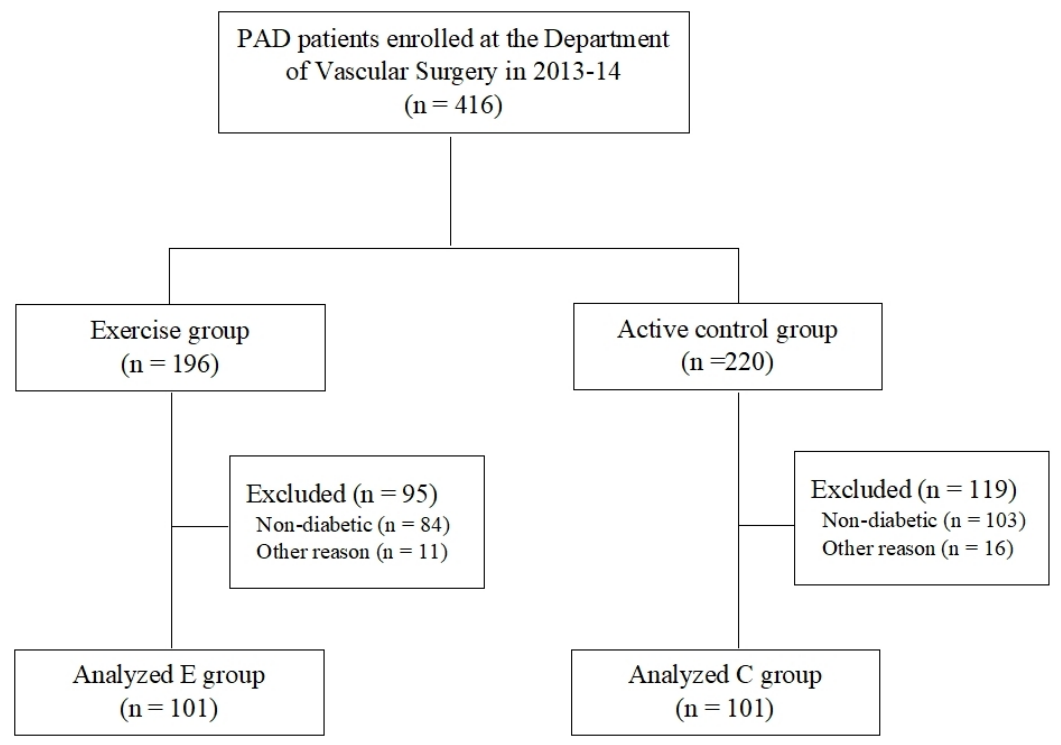

Figure 1. Flow diagram of the participants. 


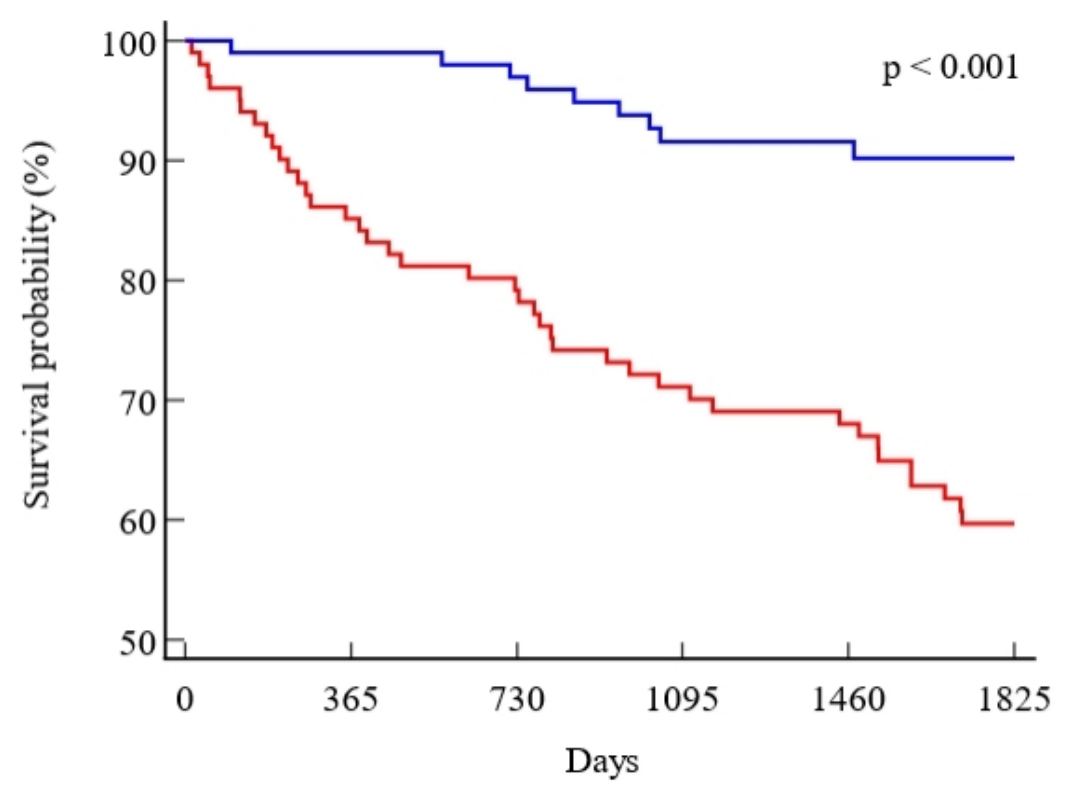

Figure 2. Kaplan-Meier survival curves in the two groups: exercise (blue) and control (red).

The multivariate analyses conducted in the two groups confirmed the results observed in the whole population, with age and comorbidities (chronic kidney disease and lung disease) as the more important risk factors for the two groups [Table 2].

\section{DISCUSSION}

The study has shown a reduction in the risk of long-term mortality and adverse vascular outcomes at five years in diabetic PAD patients who were included in the TiTo home exercise program compared to diabetic PAD patients who were simply advised to perform physical activity.

Patients with symptomatic PAD are exposed to a higher risk of the worst clinical outcomes, including major cardiovascular events and amputations, with five-year cumulative incidences of $63 \%$ and $27 \%$, respectively ${ }^{[1]}$. In the presence of diabetes, the relative risk for mortality has been found to be two- to fourfold greater than that in patients with PAD only ${ }^{[10]}$. This topic is of importance since the impact on longterm outcomes of the different treatments, including participation in exercise programs, is considered a scientific priority ${ }^{[12]}$. Peripheral revascularizations have been found to be effective in reducing those relative risks by improving walking ability ${ }^{[6]}$ alone or in combination with exercise programs, which need to be pursued to improve functional capacity, clinical outcomes, and quality of life ${ }^{[5,7]}$. While several highintensity programs have been found to be effective ${ }^{[7]}$, based not only on walking but also on cycling or armcranking $^{[2]}$, comorbidities such as musculoskeletal complaints, hypertension, peripheral neuropathy, and especially PAD may reduce the response and adherence to exercise training ${ }^{[7]}$. In addition, program availability, costs, travel, and access to supervised exercise programs are barriers that need to be considered. In the presence of these factors and considering the reduced pain perception of diabetic PAD patients who report claudication at a three-fold higher degree of muscle deoxygenation ${ }^{[32]}$, low-intensity pain-free walking, such as the TiTo program ${ }^{[19-20]}$, should be encompassed to optimize adherence and reduce the possible adverse reactions to training described previously ${ }^{[7]}$. 
Table 2. Multivariate hazard ratios ( $95 \%$ confidence interval) of the study variables for the prediction of five-year survival probability in the four patient subgroups

\begin{tabular}{lcc}
\hline & Exercise $(\boldsymbol{n}=\mathbf{1 0 1})$ & Control $(\boldsymbol{n}=\mathbf{1 0 1})$ \\
\hline Age & & \\
Male sex & \\
Smoking & \\
Hypertension & \\
Hyperlipidemia & $7.69(1.58-37.35)$ \\
Insulin-dependent diabetes & \\
Chronic kidney disease & \\
Ischemic heart disease & \\
Stroke & & \\
Pulmonary disease & \\
Neoplastic disease & \\
Age-adjusted charlson index & \\
Lower limbs revascularization & \\
Bilateral disease & \\
ABI more impaired limb & \\
ABl less impaired limb & \\
\hline
\end{tabular}

The TiTo-structured home-based program has already proved its effectiveness in large populations of patients with $\mathrm{PAD}^{[19,20]}$, with a lower relative risk for mortality, revascularizations, and all-cause hospitalizations when both considering those patients who recorded a favorable outcome after the training program $^{[24]}$ and comparing participants to non-participants of the program ${ }^{[25]}$.

This study confirmed the previous findings in a sample of the population with PAD, including only patients with diabetes. After five years, the mortality rate in the control group was $40 \%$, which is very similar to the percentage recently described ${ }^{[10]}$, but this value dropped to $10 \%$ when patients were engaged in the TiTo program, leading to an almost four-fold lower risk. Favorable data were also observed for the secondary outcomes, including PAD-related revascularizations, all-cause hospitalizations, and amputations, with hazard ratios varying from 1.5 to 2 times higher for the control group.

In a nationwide retrospective analysis after SET programs ${ }^{[33]}$, the five-year freedom from revascularizations was $83 \%$, which is similar to our study ( $85 \%$ ) but including a population of claudication patients with and without diabetes. Another study reported similar values (a survival rate of $81 \%$ in the SET group) but included only $25 \%$ diabetic patients ${ }^{[34]}$. It is interesting to observe that short bouts of in-home slow interval walking may have an impact on long-term clinical outcomes. However, the increased mobility observed in a poorly active population may represent the explanation. At the end of each week, each patient performed a total of 140 min of walking, corresponding to the amount of physical activity recommended by the guidelines $^{[2,7]}$. Several factors might also be considered. Low-moderate intensity exercise might improve nitric oxide bioavailability, decrease systemic and local oxidative stress and inflammation ${ }^{[15]}$, and induce vascular adaptations (including angiogenetic factors and lower sympathetic activation of the arteriolar tone) that are not observable following intense exercise ${ }^{[14-15]}$.

A crucial key factor in making such changes is the high adherence reported, which was favored by the characteristic of the TiTo program, such as its indoor execution (facilitating safety and avoiding any weather interference). In addition, the low intensity of exercise, which does not evoke claudication symptoms, may be another factor favoring adherence, particularly in women ${ }^{[22,35]}$. Other pain-free protocols 

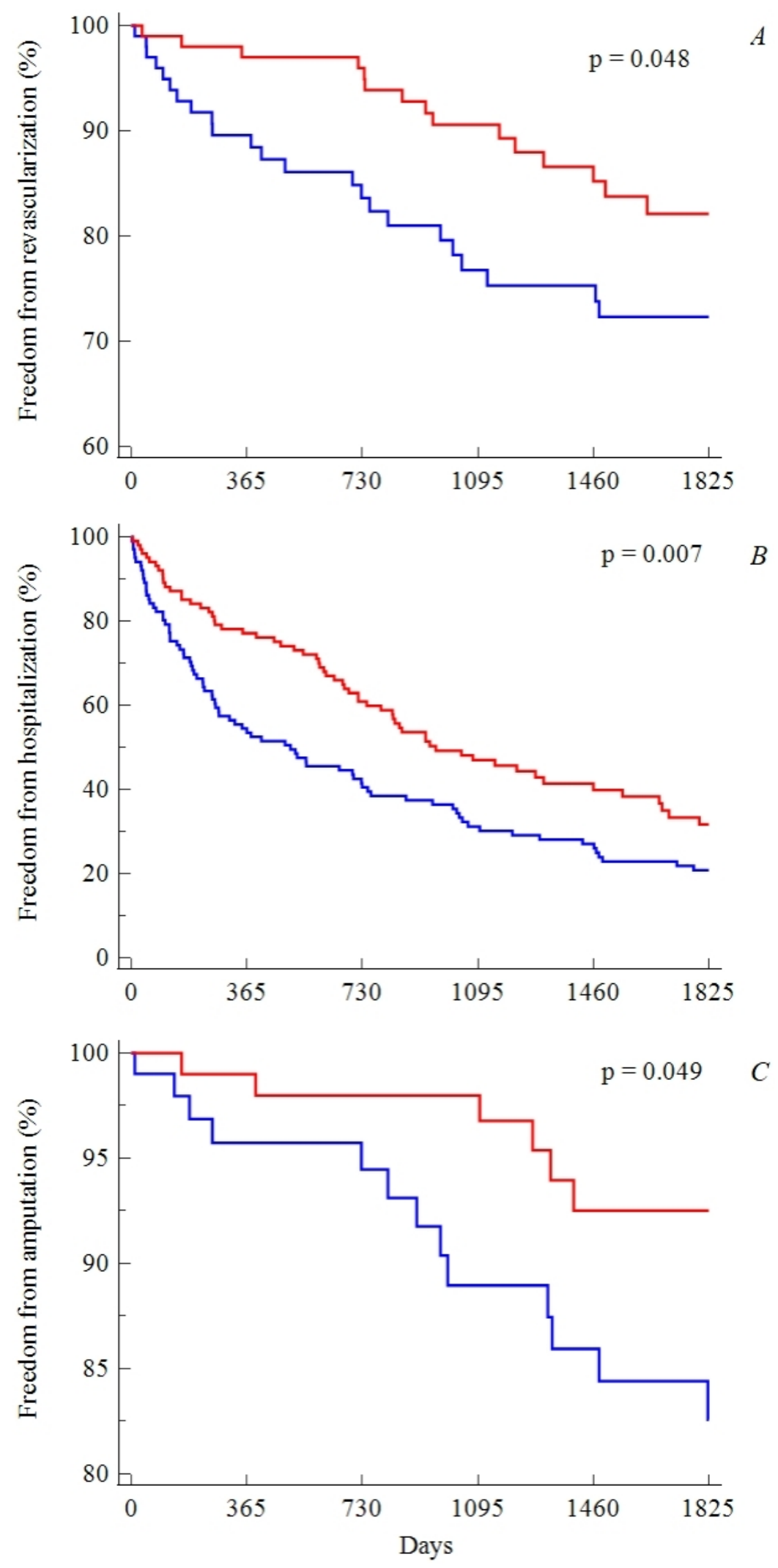

Figure 3. Kaplan-Meier curves of the two groups for: revascularization (A); all-cause hospitalizations (B); and amputation (C). The blue lines indicate exercise, while red ones indicate control. 
have been proposed and found to be effective when performed on a treadmill or using alternative forms of exercise training, such as arm-cranking or leg-cycling ergometers ${ }^{[1,36]}$. However, these protocols were supervised, with related barriers and limitations in terms of organizational issues (necessity of equipment, presence of a team at the hospital, and patients' transportation) and patient enrollment (requested ability to walk on a treadmill for least $2 \mathrm{~min}$ at $3.2 \mathrm{~km} / \mathrm{h}$ ). Moreover, these programs, unlike TiTo, may not be able to accustom the patient to ground walking and favor long-term autonomous adherence to exercise.

Several limitations affected the study, including the sample size being limited and the lack of accurate clinical data typical of a retrospective study (e.g., data on glycemic control in the observed period are missing). In addition, the amount of maintenance training suggested to patients at discharge from the exercise program was not collected. To this end, the study reflects a real-world condition (as most of this kind of studies), therefore changes in habits may have occurred randomly in both groups, since some Group E patients may have continued exercising, whereas some of the patients of Group C may have started a rehabilitation program. This fact reduces the potential implication in the results.

In conclusion, a low-intensity, structured, and home-based program was associated with a lower long-term risk of mortality, invasive vascular treatments, or amputations in patients with PAD and diabetes, even if a direct association could not be claimed. However, mobility recovery, daily exercise appointments, and painfree maintenance programs may represent favorable factors.

Properly designed exercise programs in patients affected by PAD and diabetes, as in the study presented, may have a clinical impact; however, their effectiveness needs to be confirmed in randomized trials ${ }^{[37]}$.

\section{DECLARATIONS}

\section{Acknowledgements}

We thank Dr. Corrado Francesco Professione for his contribution to data collection.

\section{Authors' contributions}

Conceived and planned the experiments: Lamberti N, Tsolaki E, Gasbarro V, Zamboni P, Straudi S, Manfredini R, Manfredini F

Carried out the experiments: Lamberti N, Straudi S, Piva G, Manfredini F

Planned and carried out the long-term data: Tsolaki E, Guerzoni F, Napoli N

Contributed to the interpretation of the results: Lamberti N, Tsolaki E, Traina L, Gasbarro V, Zamboni P, Manfredini R, Manfredini F

Took the lead in writing the manuscript: Lamberti N, Manfredini F

All authors provided critical feedback and helped shape the research, analysis and manuscript.

\section{Availability of data and materials}

The dataset of the study is available upon request to the corresponding author.

\section{Financial support and sponsorship}

None.

\section{Conflicts of interest}

All authors declared that there are no conflicts of interest. 


\section{Ethical approval and consent to participate}

The study was performed in accordance with the Declaration of Helsinki and approved by the CE-AVEC Ethics Committee (number 277/2019).

\section{Consent for publication}

Not applicable.

\section{Copyright}

(c) The Author(s) 2022.

\section{REFERENCES}

1. Agnelli G, Belch JJF, Baumgartner I, Giovas P, Hoffmann U. Morbidity and mortality associated with atherosclerotic peripheral artery disease: a systematic review. Atherosclerosis 2020;293:94-100. DOI PubMed

2. Askew CD, Parmenter B, Leicht AS, Walker PJ, Golledge J. Exercise \& Sports Science Australia (ESSA) position statement on exercise prescription for patients with peripheral arterial disease and intermittent claudication. J Sci Med Sport 2014;17:623-9. DOI PubMed

3. Gerhard-Herman MD, Gornik HL, Barrett C, et al. 2016 AHA/ACC Guideline on the management of patients with lower extremity peripheral artery disease: executive summary: a report of the American college of cardiology/American heart association task force on clinical practice guidelines. J Am Coll Cardiol 2017;69:1465-508. DOI PubMed

4. Ratliff CR, Strider D, Rovnyak V. Quality of life in individuals with peripheral arterial disease who underwent toe amputations: a descriptive, cross-sectional study. Wound Manag Prev 2019;65:34-40. PubMed

5. Aiello A, Anichini R, Brocco E, et al; Italian Society of Diabetes; Italian Society of Radiology; Italian Society of Vascular Endovascular Surgery. Treatment of peripheral arterial disease in diabetes: a consensus of the Italian Societies of Diabetes (SID, AMD), Radiology (SIRM) and Vascular Endovascular Surgery (SICVE). Nutr Metab Cardiovasc Dis 2014;24:355-69. DOI PubMed

6. Giugliano G, Perrino C, Schiano V, et al. Endovascular treatment of lower extremity arteries is associated with an improved outcome in diabetic patients affected by intermittent claudication. BMC Surg 2012;12 Suppl 1:S19. DOI PubMed PMC

7. Kemps H, Kränkel N, Dörr M, et al. Exercise training for patients with type 2 diabetes and cardiovascular disease: what to pursue and how to do it. A Position paper of the European association of preventive cardiology (EAPC). Eur J Prev Cardiol 2019;26:709-27. DOI PubMed

8. Behroozian A, Beckman JA. Microvascular disease increases amputation in patients with peripheral artery disease. Arterioscler Thromb Vasc Biol 2020;40:534-40. DOI PubMed

9. Mohammedi K, Woodward M, Hirakawa Y, et al; ADVANCE Collaborative Group. Presentations of major peripheral arterial disease and risk of major outcomes in patients with type 2 diabetes: results from the ADVANCE-ON study. Cardiovasc Diabetol 2016;15:129. DOI PubMed PMC

10. Mueller T, Hinterreiter F, Poelz W, Haltmayer M, Dieplinger B. Mortality rates at 10 years are higher in diabetic than in non-diabetic patients with chronic lower extremity peripheral arterial disease. Vasc Med 2016;21:445-52. DOI PubMed PMC

11. Arora E, Maiya AG, Devasia T, Bhat R, Kamath G. Effect of supervised exercise program on individuals in peripheral arterial disease with Type 2 diabetes mellitus - a systematic review. Curr Diabetes Rev 2020;16:248-53. DOI PubMed

12. Treat-Jacobson D, McDermott MM, Bronas UG, et al; American Heart Association Council on Peripheral Vascular Disease; Council on Quality of Care and Outcomes Research; Council on Cardiovascular and Stroke Nursing. Optimal exercise programs for patients with peripheral artery disease: a scientific statement from the American heart association. Circulation 2019;139:e10-33. DOI PubMed

13. Kim K, Anderson EM, Scali ST, Ryan TE. Skeletal muscle mitochondrial dysfunction and oxidative stress in peripheral arterial disease: a unifying mechanism and therapeutic target. Antioxidants (Basel) 2020;9:E1304. DOI PubMed PMC

14. Andrade-Lima A, da Silva Junior N, Chehuen M, et al. Local and systemic inflammation and oxidative stress after a single bout of maximal walking in patients with symptomatic peripheral artery disease. J Cardiovasc Nurs 2020. DOI PubMed

15. Andrade-Lima A, Silva Junior N, Chehuen M, et al. Walking training improves systemic and local pathophysiological processes in intermittent claudication. Eur J Vasc Endovasc Surg 2021;61:954-63. DOI PubMed

16. Pipinos II, Judge AR, Selsby JT, et al. The myopathy of peripheral arterial occlusive disease: part 1. Functional and histomorphological changes and evidence for mitochondrial dysfunction. Vasc Endovascular Surg 2007;41:481-9. DOI PubMed

17. Lyu X, Li S, Peng S, Cai H, Liu G, Ran X. Intensive walking exercise for lower extremity peripheral arterial disease: a systematic review and meta-analysis. J Diabetes 2016;8:363-77. DOI PubMed

18. Manfredini F, Conconi F, Malagoni AM, et al. Training guided by pain threshold speed. Effects of a home-based program on claudication. Int Angiol 2004;23:379-87. PubMed

19. Manfredini F, Malagoni AM, Mascoli F, et al. Training rather than walking: the test in -train out program for home-based rehabilitation in peripheral arteriopathy. Circ J 2008;72:946-52. DOI PubMed

20. Malagoni AM, Vagnoni E, Felisatti M, et al. Evaluation of patient compliance, quality of life impact and cost-effectiveness of a "test in-train out" exercise-based rehabilitation program for patients with intermittent claudication. Circ J 2011;75:2128-34. DOI PubMed

21. Lamberti N, Straudi S, Lissia E, et al. Home-based exercise for elderly patients with intermittent claudication limited by osteoarticular disorders - feasibility and effectiveness of a low-intensity programme. Vasa 2018;47:227-34. DOI PubMed 
22. Manfredini R, Lamberti N, Manfredini F, et al. Gender differences in outcomes following a pain-free, home-based exercise program for claudication. J Womens Health (Larchmt) 2019;28:1313-21. DOI PubMed PMC

23. Lamberti N, Malagoni AM, Ficarra V, et al. Structured home-based exercise versus invasive treatment: a mission impossible? Angiology 2016;67:772-80. DOI PubMed

24. Manfredini F, Lamberti N, Guerzoni F, et al. Rehabilitative exercise reduced the impact of peripheral artery disease on vascular outcomes in elderly patients with claudication: a three-year single center retrospective study. J Clin Med 2019;8:E210. DOI PubMed PMC

25. Lamberti N, López-Soto PJ, Guerzoni F, et al. Changes in exercise capacity and risk of all-cause mortality in patients with peripheral artery disease: a 10-year retrospective cohort study. Intern Emerg Med 2020;15:289-98. DOI PubMed

26. Lamberti N, Straudi S, Malagoni AM, et al. Effects of low-intensity endurance and resistance training on mobility in chronic stroke survivors: a pilot randomized controlled study. Eur J Phys Rehabil Med 2017;53:228-39. DOI PubMed

27. Malagoni AM, Cavazza S, Ferraresi G, Grassi G, Felisatti M, et al. Effects of a "test in-train out" walking program versus supervised standard rehabilitation in chronic stroke patients: a feasibility and pilot randomized study. Eur J Phys Rehabil Med 2016;52:279-87. PubMed

28. Malagoni AM, Catizone L, Mandini S, et al. Acute and long-term effects of an exercise program for dialysis patients prescribed in hospital and performed at home. J Nephrol 2008;21:871-8. PubMed

29. Manfredini F, Mallamaci F, D'Arrigo G, et al. Exercise in patients on dialysis: a multicenter, randomized clinical trial. $J$ Am Soc Nephrol 2017;28:1259-68. DOI PubMed PMC

30. Lamberti N, Straudi S, Donadi M, Tanaka H, Basaglia N, Manfredini F. Effectiveness of blood flow-restricted slow walking on mobility in severe multiple sclerosis: a pilot randomized trial. Scand J Med Sci Sports 2020;30:1999-2009. DOI PubMed

31. Norgren L, Hiatt WR, Dormandy JA, Nehler MR, Harris KA, Fowkes FG; TASC II Working Group. Inter-society consensus for the management of peripheral arterial disease (TASC II). J Vasc Surg 2007;45 Suppl S:S5-67. DOI PubMed

32. Manfredini F, Lamberti N, Malagoni AM, et al. Reliability of the vascular claudication reporting in diabetic patients with peripheral arterial disease: a study with near-infrared spectroscopy. Angiology 2015;66:365-74. DOI PubMed

33. Jansen SCP, van Nistelrooij LPJ, Scheltinga MRM, Rouwet EV, Teijink JAW, Vahl AC. Successful implementation of the exercise first approach for intermittent claudication in the netherlands is associated with few lower limb revascularisations. Eur J Vasc Endovasc Surg 2020;60:881-7. DOI PubMed

34. Bouwens E, Klaphake S, Weststrate KJ, et al. Supervised exercise therapy and revascularization: single-center experience of intermittent claudication management. Vasc Med 2019;24:208-15. DOI PubMed PMC

35. Dipnarine K, Barak S, Martinez CA, Carmeli E, Stopka CB. Pain-free treadmill exercise for patients with intermittent claudication: are there gender differences? Vascular 2016;24:304-14. DOI PubMed

36. Mika P, Konik A, Januszek R, et al. Comparison of two treadmill training programs on walking ability and endothelial function in intermittent claudication. Int J Cardiol 2013;168:838-42. DOI PubMed

37. Manfredini F, Lamberti N, Traina L, et al. Effectiveness of home-based pain-free exercise versus walking advice in patients with peripheral artery disease: a randomized controlled trial. Methods Protoc 2021;4:29. DOI PubMed PMC 